\title{
Interventions to support early childhood development in times of COVID-19: A Systematic Review
}

\author{
Komal Abdul Rahim ${ }^{1}$, Shelina Bhamani ${ }^{2}$ and Zohra S Lassi ${ }^{3 *}$ \\ ${ }^{1}$ Cardiology Medicine, The Aga Khan University, Pakistan \\ ${ }^{2}$ Department of Obstetrics and Gynecology, The Aga Khan University, Pakistan \\ ${ }^{3}$ Robinson Research Institute, the University of Adelaide, Australia
}

\begin{abstract}
Background: COVID-19 which has come as an emerging disease was declared a pandemic by the World Health Organization in March 2020. In an attempt to limit the spread of this virus, strict measures were taken amongst which closure of schools and child care facilities were also implemented. This impacted the holistic well-being of the children.

Objective: This systematic review aimed at identifying early childhood development (ECD) interventions targeted to children 0-8 years of age conducted and reported during the COVID-19 pandemic in low and middle-income countries, and also to identify the barriers and facilitators to taking up ECD activities during the COVID-19 pandemic.

Methodology: This systematic review considered all published and pre-print studies published between 31 Dec 2019 and 31 Dec 2020 and followed PRISMA guidelines for the conduct of the systematic review. Databases such as MEDLINE, Embase, ERIC, CINAHL, the Cochrane Central Register of Controlled Trials (CENTRAL), Google Scholar, and the WHO COVID-19 database were searched.
\end{abstract}

Result: Zero studies were included at the end of the full-text screening. Few papers were fitting well with the scope of the paper; however, those papers were not fitting the study design. The majority of those papers were position papers and opinion papers.

Conclusion: This empty review highlighted a major research gap in the literature. There were no studies conducted to address the interventions taken place in the pandemic to support ECD. There is a dire need to address the issue by conducting more quantitative and qualitative studies.

\section{Introduction}

COVID-19 came as an emerging disease that has transformed the daily living of the people to a greater extent, and the functions around the globe. Many measures have been taken by states around the world to limit the spread of viral illness. One of the measures was seen in the form of nationwide lockdown and closures of schools including the child healthcare facility; this has impacted the overall health of children especially those in the age range of $0-8$ years [1]. These measures have disrupted the daily living of the children who stays at home and being dependent on the caregivers for their nurturing. This includes holistic needs physical, cognitive, and psycho-social aspects of growth and development. These issues, though have been seen in children across the globe, the results are amplified in unprivileged areas such as those living in low-and-middle-income countries (LMICs).

The reports concluded before the pandemic highlighted that there were $43 \%$ of children worldwide who were at higher risk of not reaching their core developmental milestones under the age of 5 years [2]. After the closure of schools, particularly in LMICs, the younger children had no proper access to learning materials (either in-person or online) that may have influenced their gross and fine motor skills. Furthermore, the nationwide lockdown also led to the closure of public areas such as parks and other social gatherings areas that limited the movement of children out of their homes; this greatly affected the psycho-social health, peer interaction, social support, and connections that are vital for cognitive stimulation outside the home. This greatly affected the families who were part of the inclusive childcare program; they become deprived of the nutritional requirement including meals and supplements, and other facilities that were provided to them as part of the Early Childhood Development (ECD) organizations and agencies [3]. Thus, the global agencies need to boast ECD interventions to meet the requirement of both the children and their families. Amongst the many international frameworks, the nurturing care framework is an internationally recognized framework encompassing 5 major components for the ECD. As per the highlighted components, the children aged 0-8 years should have access to health, learning, and stimulation, and protection, and responsive caregiving [4] (Figure 1).

The policy statement provided by the United Nations (UN) highlighted financial crises and economic distress as being a strong predictor during the pandemic, which will potentially affect 42 62 million children to moderate and severe poverty index [5]. This may likewise impact the population to face food security while also increasing the expenditure on healthcare. Unemployment and financial crises can lead to internal displacement, migration, affecting family dynamics as a whole that can eventually lead to divorce, separation,

${ }^{\star}$ Correspondence to: Zohra S Lassi, Robinson Research Institute, the University of Adelaide, Australia, E-mail: Zohra.lassi@adelaide.edu.au

Key words: COVID-19, early childhood development, childhood interventions

Received: June 14, 2021; Accepted: June 25, 2021; Published: June 28, 2021 


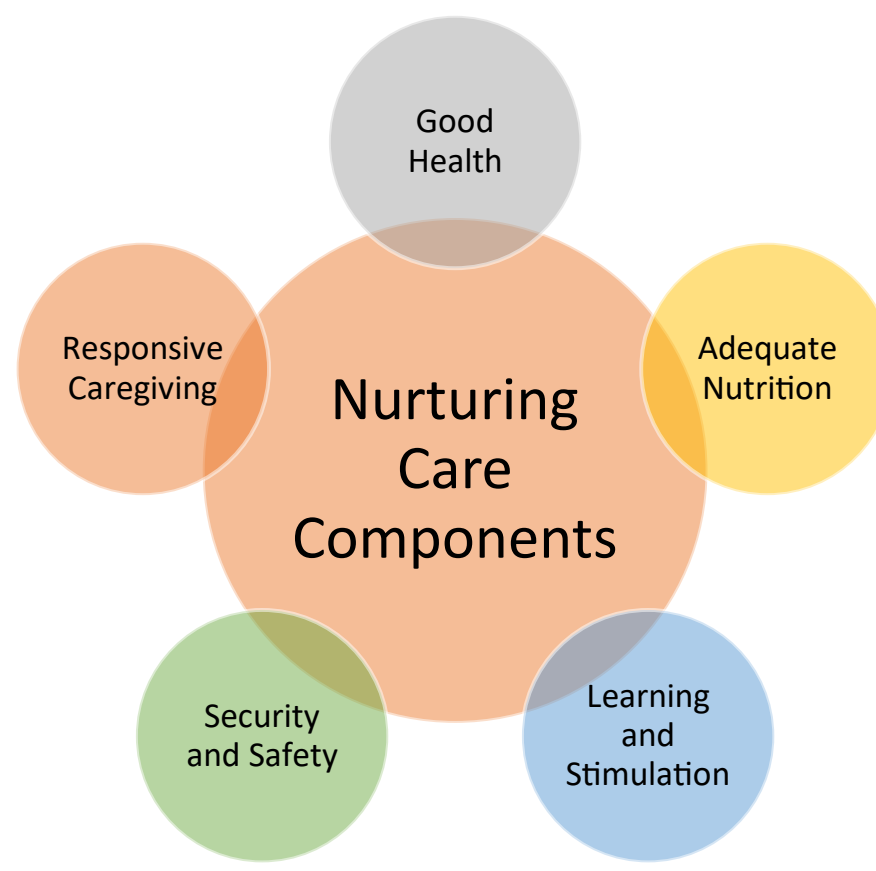

Figure 1. Nurturing Care Components of the Early Childhood Development Adapted from the World Health Organization 2017 [4]

and domestic violence. These disrupted family dynamics directly affect the psychological health and overall development of young children. In addition to this, rising poverty, the decline in food insecurity, increased stress, and lack of utilization of healthcare, continue affecting the health of young children in a negative way on a global scale [6]. This not only affects individual children but can lead to transgenerational impact causing epigenetic and psychological alterations. It is thus significant to give more attention to ECD interventions during the COVID-19 pandemic.

Given the importance of ECD, global organizations have tried their best to ensure that children around the globe get support during this pandemic. There are many healthcare agencies, educational institutions, and civil society organizations that are already working during the pandemic for the nutritional needs, mental health, and health needs of younger children aged 0-8 years [7]. During the pandemic, the key stakeholders have pulled their efforts in giving knowledge, raising awareness, and sensitizing communities for the severity of the virus. The information commitment is additionally optimized and sped up to share the discoveries internationally of what deals with the ground. This didn't give adequate time and center to the interventionist to share their arranged mediations and their effect. There have been introductory discoveries on the adequacy of help gave through different mediations yet there is an absence of information on ECD interventions detailed from LMICs [8]. A preliminary search carried out for this review in different databases informed the similar stand [9].

The varied interventions planned during the pandemic might have bolstered the components highlighted in Figure 1. Studies usually conclude that providing interventions in settings that have limited access to the resources, if, given the opportunities to these younger children to thrive, the is an increased probability that the outcomes get improved. This systematic review has assessed ECD interventions provided based on nurturing care framework (Figure 1) [4] and their impact on child's nurturing outcomes in the times of the COVID-19.
The findings from this systematic review will benefit the parents/ caregivers and ECD practitioners working in LMICs. By exploring different interventions carried out in LMICs in times of COVID-19 that address any one component of the nurturing care framework will enable practitioners and care service providers in planning interventions to support the developmental health of young children. This will benefit in planning relevant child development intervention models, assessing their needs, and strategizing a way forward for programs that may be planned post-pandemic for young children and their families. Exploring interventions and knowledge gaps will provide insights to the care delivering agencies to ensure that children's needs are streamlined in times of crisis and beyond. In addition, this review will also provide reflections to the academia to plan a relevant investigation to explore the data trends and strategize their research work in accordance to inform knowledge.

This systematic review aimed to compile and assess the effectiveness of the initiatives undertaken by local, national, and international authorities to address the ECD needs of children living in LMICs during the COVID-19 pandemic. The following review questions were considered:

1. What are the different ECD interventions and their effectiveness during the COVID-19 pandemic in LMICs?

2. What are the barriers and facilitators to taking up ECD activities during the COVID-19 pandemic in LMICs?

\section{Methods}

The systematic review has followed the guidelines proposed by Preferred Reporting Items for Systematic Reviews and Meta-Analyses (PRISMA) [3] (Annex 1). This systematic review has been registered in the PROSPERO: CRD42020202541 [10].

\section{Types of Studies}

The review included interventional, observational, and qualitative studies. All types of interventions were included regardless of their setting i.e. community, clinical, etc. Interventions conducted in the LMICs by programmatic agencies, non-governmental organizations, governmental organizations, community-based organizations, academia, and independent researchers were considered.

\section{Participants}

The review considered studies that included children 0 to 8 years and their caregivers, living in LMICs. All children were considered, regardless of their health condition. The list of the LMICs was considered as given by the World Bank in the year 2020 [11].

\section{Interventions}

This review considered ECD interventions implemented during the COVID-19. ECD interventions are defined as programs that focus on either any one, or all aspects of the nurturing care framework, i.e., health, nutrition, responsive caregiving, security and safety, and learning and stimulation.

\section{Comparator}

The review included the studies that have compared ECD intervention with no intervention or standard of care intervention. 


\section{Outcomes}

The review considered studies that reported the outcomes that focused on the core components of nurturing care framework. These indicators include the health of young children ages 0-8 years which include physical and mental health, education provision, nutrition, and health supplementation provision and access, protection services for children and their families, and policy interventions which are a core part of nurturing care framework. We also aim to assess the facilitators and barriers of taking up ECD interventions during the COVID-19 pandemic.

The search strategy was prepared with the help of a librarian from the Aga Khan University using the Medical Subject Headings (MeSH) terms along with key terms (Annex 2). The databases such as MEDLINE, Embase, ERIC, CINAHL, the Cochrane Central Register of Controlled Trials (CENTRAL), Google Scholar, and the WHO COVID-19 were used to search the potential papers. The studies which are published in the English language between 31 Dec 2019 to $31 \mathrm{Dec}$ 2020 were considered.

After running the search strategy, all the identified studies were saved into the EndNote library X8.1 and the duplicates were removed. These files were then imported on COVIDENCE [12] for the initial step of title and abstract screening by two independent reviewers (KAR and SB) in conjunction with the inclusion criteria of the review. The conflicts arising while screening was resolved by an expert reviewer from the team (ZSL). The studies which qualified in the title and abstract screening were then moved to the next stage of full-text screening. The conflicts during this phase were resolved by an expert reviewer (ZS). Studies excluded at this stage were marked with the reason for exclusion and presented in a logical flow diagram known as Preferred Reporting Items for Systematic Reviews and Meta-Analyses (PRISMA) flow diagram.

Two review authors independently extracted the data and assess the methodological quality of included studies. Any disagreements were resolved by an expert reviewer from the team. The data extracted from these studies included the basic study characteristics, details of the intervention, and the details of the outcome. A preliminary data extraction table is attached in appendix 3 . The methodological quality of intervention studies was assessed using the ROBINS-I checklist [13] (Annex 3 and 4), observational studies using NHLBI criteria (Annex 5) [14], and the qualitative studies using CASP criteria (Annex 6) [15].

We initially aim to use Review Manager [16] for the meta-analysis; however, the review did not identify any eligible study for inclusion. Categorical outcomes were assessed using the relative risk with $95 \%$ confidence intervals (CI) and continuous outcomes were assessed using the mean difference with 95\% CIs. Furthermore, barriers to the implementation of ECD interventions were recorded through a qualitative synthesis.

\section{Results}

A total of 573 articles were retrieved from the databases and the grey literature including Google Scholar and institutional websites. After removing the duplicates, 559 articles were unique which entered the title and abstract screening stage. After the screening performed by two independent reviewers, 527 articles were excluded, and a total of 32 papers entered the next stage i.e. the full-text screening. Although few of the studies did mention the ECD interventions and recommended some measures to improve the ECD outcomes during the time of the COVID-19 pandemic, however, none of these met the inclusion criteria. As a result, all of the studies at this stage were excluded. The reasons for exclusion are mentioned in annex 7 . Thus, there were 0 studies included in the final data extraction (Figure 2).

\section{Discussion}

The primary aim of this study was to systematically identify the interventions related to ECD during the pandemic for children aged 0-8 years old. Unfortunately, none of the studies met the inclusion criteria of the review. We found 5 papers that do account for the ECD interventions in some way or the other; however, those were discussion papers [17], position papers [18,19], testimony [20], or commentaries [21]. ECD interventions are paramount in the development of children during the initial years of life. The absence of a study shows a major research gap.

The closure of the schools in an attempt to contain the spread of the virus has led to great educational loss amongst the children, especially in their early years. As per the statement of the United Nations (UN) Secretary, the COVID-19 pandemic has led to an education loss in history and has greatly affected learning which can stretch up to one generation [22]. To mitigate the educational and learning loss amongst the children, four recommendations have been proposed by the UN: 1) identifying the ways to contain the spread of the virus and look for the possibility of school re-opening, 2) invest more to provide finance in the education and coordinate for its impact, 3) strengthening education system to a point to build resilience and trying to achieve the sustainable development goals, 4) and reflect on the educational system and amplify positive trends in the teaching and learning during the pandemic [22].

United Nations International Children's Emergency Fund (UNICEF) has also acknowledged the drastic effects of COVID-19 on ECD. They highlighted the need for an integrated approach to responding to emergencies of ECD [23]. In addition to the integrated and intersectoral interventions, it is also important to identify the response and recovery strategies with partnered organizations for the holistic development of children aged $0-8$ years. They proposed a three-tier approach for COVID-19 response and recovery alongside the recommendations (Figure 3).

United Nations Educational, Scientific, and Cultural Organization (UNESCO) arranged a webinar on COVID-19 where the key issues relating to the ECD and COVID-19 were discussed and a call to action was proposed. UNESCO highlighted the impact of school closure on the overall health, nutrition, education, social protection, and social and emotional nurturing of the children posing a great threat to the

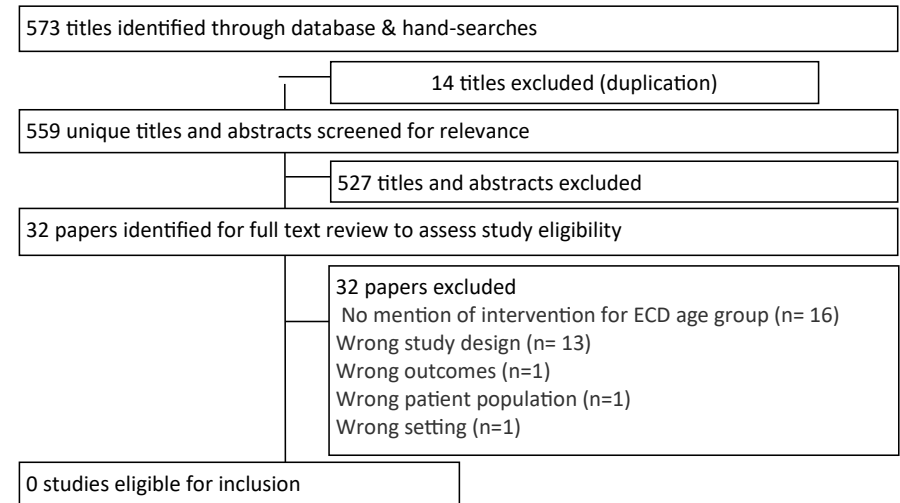

Figure 2. PRISMA Flow Diagram 


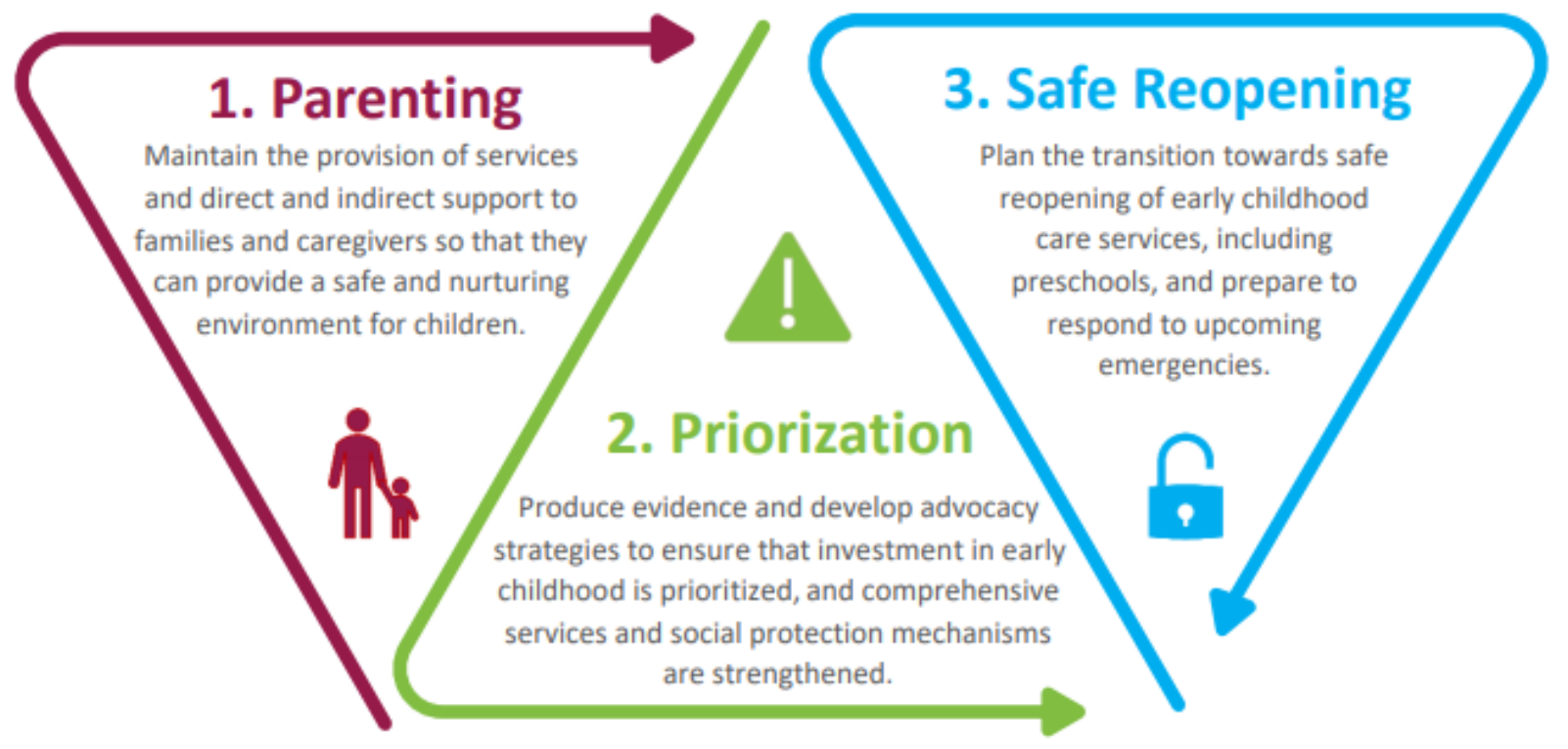

Figure 3. UNICEF three-tier model for ECD interventions [23]

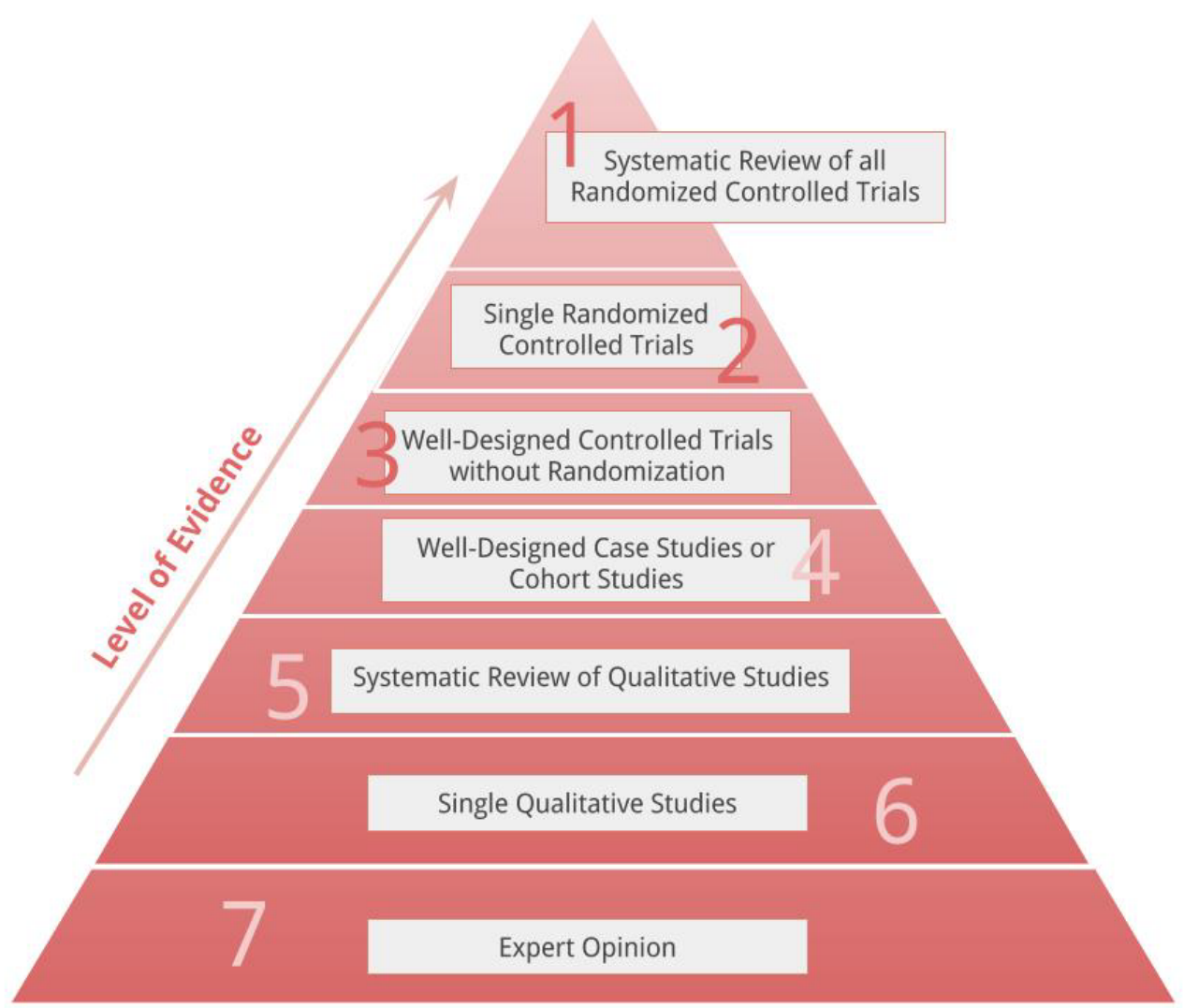

Figure 4. Pyramid of Evidence [28] 
development of children aged 0-8 years old [24]. The negligence of the ECD was persistent in the pre-COVID-19 times too; based on all the emergencies and discussion roaming around the ECD, two actions were proposed to mitigate the problem: 1) training of around 200,000 early childhood educators using various online platforms, and 2) consensus on the global strategy for the Early Childhood Care and Education through effective collaboration, engaging in fruitful dialogues and building alliances [24].

As part of the assessment and strategies planning during the pandemic in Pakistan, the stakeholders highlighted the need for addressing ECD; the report stated "ECD interventions should be prioritized to address children's needs in terms of health, nutrition, cognitive development, psychosocial development, and language" [25].

\section{Limitations of the Study}

Given the importance of the most pressing topic i.e. ECD interventions in COVID-19 times, the inclusion of 0 studies for data synthesis is the major limitation of the study. The potential purpose of this review was also to meta-analyze and pool the data for showing its effectiveness and making recommendations.

Secondly, all the papers which were identified before the data extraction stage were either position papers, opinions, or editorials. These are never included to conclude any evidence. In the pyramid of evidence, these study designs are located at the base of the hierarchy i.e. level 7 (Figure 4). At the base of the pyramid, the study designs provide the lowest level of evidence and a great risk of bias [26]. This is because the information from these studies is unfiltered and is coming from the perspective of the writer without following the research process [27].

\section{Implications and Conclusion}

We conclude this systematic review is empty. It is a common perception that the inclusion of 0 studies in the data synthesis of a systematic review does not provide any significant information which can be a help for the clinicians and the decision and policy-makers [29]. However, literature also stress the importance of systematic reviews with 0 inclusions; this is a finding in itself that there is no evidence aligned with the research question of the systematic review [30]. In these instances, empty reviews can be of great importance. Also, this will help guide the clinicians and the researchers to develop and conduct some clinical trials or other appropriate research to answer the research question $[29,30]$.

In conclusion, ECD is important for the overall functionality of children. However, the COVID-19 pandemic became a great hindrance to effective ECD. The closure of the schools and the containment strategies imposed by the government during the pandemic had affected the educational, nutritional, mental, and emotional wellbeing of the children. To date, many studies have been conducted to work on the ECD interventions, however, none of the studies have been conducted to propose ECD interventions and their effects on the overall well-being of the children. These results are conclusive based on the 0 studies included in this systematic review. This highlights a major research gap in the evidence-based literature; thus, there is a need to conduct studies that proposes ECD interventions in the COVID-19 and provide conclusive results in this area for the better growth and development of children aged $0-8$ years.

\section{Conflict of Interest}

There is no conflict of interest, and this is not a funded project.

\section{References}

1. Phelps C, Sperry LL (2020) Children and the COVID-19 pandemic. Psychol Trauma 12: S73-S75. [Crossref]

2. Brewer TE, Caldwell FT, Patterson RM, Flanigan WJ (1972) Indwelling peritonea (Tenckhoff) dialysis catheter. Experience with 24 patients. JAMA 219: 1011-1015. [Crossref]

3. Moher D, Liberati A, Tetzlaff J, Altman DG, Group TP (2009) Preferred Reporting Items for Systematic Reviews and Meta-Analyses: The PRISMA Statement. PLOS Med 6: e1000097. [Crossref]

4. WHO (2021) Nurturing care for early childhood development: Linking survive and thrive to transform health and human potential. World Health Organization.

5. Cheng TL, Moon M, Artman M, Pediatric Policy Council (2020) Shoring up the safety net for children in the COVID-19 pandemic. Pediatr Res 88: 349-351. [Crossref]

6. Sinha IP, Lee AR, Bennett D, McGeehan L, Abrams EM, et al. (2020) Child poverty, food insecurity, and respiratory health during the COVID-19 pandemic. Lancet Respir Med 8: 762-763. [Crossref]

7. Suleman S, Ratnani Y, Stockley K, Jetty R, Smart K, et al. (2020) Supporting children and youth during the COVID-19 pandemic and beyond: A rights-centred approach. Paediatr Child Health 25: 333-336. [Crossref]

8. Akseer N, Kandru G, Keats EC, Bhutta ZA (2020) COVID-19 pandemic and mitigation strategies: implications for maternal and child health and nutrition. The American Journal of Clinical Nutrition 112: 251-256.

9. Roelen K, Ackley C, Boyce P, Farina N, Ripoll S (2020) COVID-19 in LMICs: The Need to Place Stigma Front and Centre to Its Response. Eur J Dev Res 21: 1-21. [Crossref]

10. https://www.crd.york.ac.uk/prospero/display_record.php?RecordID=202541

11. World Bank (2020) World Bank Country and Lending Groups - World Bank Data Help Desk.

12. Covidence (2019) Covidence - Better systematic review management

13. Slim K, Nini E, Forestier D, Kwiatkowski F, Panis Y, et al. (2003) Methodological index for non-randomized studies (minors): development and validation of a new instrument. ANZ J Surg 73: 712-716. [Crossref]

14. https://www.nhlbi.nih.gov/health-topics/study-quality-assessment-tools

15. https://casp-uk.net/casp-tools-checklists/

16. https://training.cochrane.org/online-learning/core-software-cochrane-reviews/revman

17. Guan H, Okely AD, Aguilar-Farias N, Cruz B del P, Draper CE, et al. (2020) Promoting healthy movement behaviours among children during the COVID-19 pandemic. Lancet Child Adolesc Health 4: 416-418. [Crossref]

18. OMEP Executive Committee, World Organisation for Early Childhood Education (2020) OMEP Position Paper: Early Childhood Education and Care in the Time of COVID-19. Int J Early Child 3: 1-10. [Crossref]

19. O'Connor DB, Aggleton JP, Chakrabarti B, Cooper CL, Creswell C, et al. (2020) Research priorities for the COVID-19 pandemic and beyond: A call to action for psychological science. Br J Psychol 111: 603-629. [Crossref]

20. Cohen D (2020) [Appreciating COVID-19 as a child and adolescent psychiatrist on the move]. Encephale 46: S99-S106. [Crossref]

21. Choi KR, Records K, Low LK, Alhusen JL, Kenner C, et al. (2020) Promotion of Maternal-Infant Mental Health and Trauma-Informed Care During the COVID-19 Pandemic. J Obstet Gynecol Neonatal Nurs 49: 409-415. [Crossref]

22. UNESCO (2020) COVID-19 Policy Brief: UN Secretary-General warns of education catastrophe. IIEP-UNESCO.

23. UNICEF (2020) Strategic Guidelines to Prioritize Early Childhood Development in the COVID-19 Response.

24. UNESCO (2021) New drive to protect early childhood education in the context of the COVID-19 crisis [Internet]. UNESCO.

25. UNDP (2020) COVID-19 Pakistan: Socio-Economic Framework | UNDP in Pakistan [Internet]. UNDP.

26. Burns PB, Rohrich RJ, Chung KC (2012) The Levels of Evidence and their role in Evidence-Based Medicine. Plast Reconstr Surg 128: 305-310. [Crossref] 
Rahim KA (2021) Interventions to support early childhood development in times of COVID-19: A Systematic Review

27. Mulimani PS (2017) Evidence-based practice and the evidence pyramid: A 21st century orthodontic odyssey. Am J Orthod Dentofacial Orthop 152: 1-8. [Crossref]

28. Murad MH, Asi N, Alsawas M, Alahdab F (2016) New evidence pyramid. Evid Based Med 21: 125-127. [Crossref]
29. Yaffe J, Montgomery P, Hopewell S, Shepard LD (2012) Empty Reviews: A Description and Consideration of Cochrane Systematic Reviews with No Included Studies. PLoS One 7: e36626 [Crossref]

30. Lang A, Edwards N, Fleiszer A (2007) Empty systematic reviews: hidden perils and lessons learned. J Clin Epidemiol 60: 595-597. [Crossref]

Copyright: (C2021 Rahim KA. This is an open-access article distributed under the terms of the Creative Commons Attribution License, which permits unrestricted use, distribution, and reproduction in any medium, provided the original author and source are credited. 